\title{
Cilostazol Suppresses IL-23 Production in Human Dendritic Cells via an AMPK- Dependent Pathway
}

\author{
Quanxing Shi ${ }^{a}$ Zhao Yin ${ }^{a}$ Peilin Liu ${ }^{a}$ Bei Zhao ${ }^{a}$ Zhong Zhang ${ }^{a}$ Shuai Mao \\ Tengfei Weia Mengmeng Rao ${ }^{a}$ Liguo Zhang ${ }^{b}$ Shouli Wangca \\ aDepartment of Cardiology, The 306th Hospital of Chinese PLA, Beijing, ${ }^{b}$ CAS Key Laboratory of \\ Infection and Immunity, Institute of Biophysics, Chinese Academy of Sciences, Beijing, ${ }^{~} \mathrm{Clinical}$ College \\ of the 306th Hospital of Chinese PLA, AnHui Medical University, Hefei, China
}

\section{Key Words}

Cilostazol • Dendritic cell $・$ IL-23 • AMPK • TLR

\begin{abstract}
Background/Aims: Cilostazol has been previously demonstrated to inhibit IL-23 production in human synovial macrophages via a RhoA/ROCK-dependent pathway. However, whether cilostazol affects IL-23 production in human dendritic cells remains largely unknown. The present study was designed to investigate this question and elucidate the possible underlying mechanisms. Methods: Human monocyte-derived dendritic cells (mo-DCs) were pretreated with or without cilostazol and then incubated with zymosan. Enzyme-linked immunosorbent assay (ELISA) and real time PCR analyses were used to measure IL-23 protein expression and RNA levels, respectively, whereas Western blotting was used to measure the expression and phosphorylation level of AMPK. Results: Our results demonstrated that cilostazol suppressed zymosan-induced IL-23 protein production in a concentration dependent manner without affecting dendritic cell viability. In addition, it was found that cilostazol suppressed the expression of the p19 and p40 subunits of IL-23. Moreover, cilostazol mimicked the effect of the AMPK agonist A-769662, as demonstrated by the fact that IL-23 production was also inhibited by A-769662, and the effect of cilostazol on IL-23 production was blocked by the AMPK antagonist Compound C. More importantly, Western blotting demonstrated that cilostazol led to an increased phosphorylation of AMPK. Conclusion: Collectively, our data suggest that cilostazol inhibits the production of IL-23 in human mo-DCs, potentially via the activation of AMPK. This suggests that cilostazol could be an effective anti-inflammatory agent in IL-23- and dendritic cell-related diseases.
\end{abstract}




\section{Introduction}

Cilostazol, a phosphodiesterase type 3 (PDE3) inhibitor, has been widely used to alleviate intermittent claudication in individuals with peripheral vascular disease [1]. Its mechanism of action has been attributed to its vasodilatory effect and the inhibition of platelet aggregation [2]. By acting through PDE3, cilostazol suppresses the degradation of cAMP, resulting in increased intracellular cAMP concentrations and corresponding downstream events. While the inhibition of platelet aggregation by cilostazol has been accepted for decades, its modulatory role in the inflammatory cascade warrants further study.

Dendritic cells (DCs), important antigen presenting cells, act as a bridge between innate and adaptive immune responses [3,4]. When exposed to microbial infections, dendritic cells rapidly secrete IL-23 [5, 6]. IL-23 is a member of the IL-12 superfamily, which is a heterodimer composed of IL-23 p19 and p40. IL-23 is a proinflammatory cytokine that is known to be produced by activated antigen-presenting cells, including DCs and macrophages. In addition, IL-23 has been demonstrated to play important immune regulatory roles in the body, including inducing T helper 17 cells (Th17) differentiation and promoting IL-22 production. Studies showed that IL-23 is involved in many autoimmune diseases, such as rheumatoid arthritis (RA) [7], and regulation of IL-23 pathway was demonstrated to play a role in the improvement of psoriasis [8]. More importantly, Subramanian et al. demonstrated IL-23's involvement in the development of cardiovascular diseases, such as atherosclerosis [9] and Hu et al. demonstrated IL-23's involvement in myocardial ischemia and reperfusion (I/R) injury [10]. Therefore, IL-23 may serve as a pivotal target for treating inflammatory and cardiovascular diseases.

Many studies [11-16] have focused on the pleiotropic effects of cilostazol (beyond its anti-platelet effect); however, the mechanisms underlying the anti-inflammatory effect of cilostazol are not always the same and depend on the cell type involved. In view of the important role of IL-23 in inflammatory diseases and cardiovascular diseases, it is crucial to investigate the effect of cilostazol on IL-23 production. Only one study has demonstrated that cilostazol inhibits IL-23 production in human synovial macrophages [16], but there has not been any investigation of cilostazol's effect on IL-23 production in DCs. Given that DCs are important IL-23-producing cells in humans, we present our findings on the effects of cilostazol on IL-23 production in human mo-DCs and then explore the possible underlying mechanisms.

\section{Materials and Methods}

\section{Reagents and Antibodies}

The following reagents were used: zymosan (Sigma-Aldrich), cilostazol (Sigma-Aldrich), DMSO (SigmaAldrich), A-769662 (Santa Cruz) and Compound C (Medchemexpress). AMPK (adenosine 5'-monophosphate (AMP)-activated protein kinase) and p-AMPK antibodies were from Cell Signaling Technology. Tubulin mouse monoclonal antibodies were from Beyotime Biotechnology (Shanghai, China). Human GM-CSF and IL-4 were from Peprotech Inc.

\section{Cell Cultures and Transfection}

Peripheral blood mononuclear cells (PBMCs) were obtained from healthy human donors and were isolated via Ficoll-Paque density gradient centrifugation. PBMCs were resuspended in RPMI 1640 medium containing $20 \mathrm{mM}$ HEPES, $100 \mathrm{U} / \mathrm{ml}$ penicillin/streptomycin, $2 \mathrm{mM}$ L-glutamine, 1\% nonessential amino acids, and 10\% heat-inactivated fetal bovine serum (Gibco, USA). Cells were then plated in 10-cm dishes for $2 \mathrm{~h}$ in an incubator with $5 \% \mathrm{CO}_{2}$, and monocytes were obtained by discarding the non-adherent cells. The purity and viability of CD14+ cells were consistently over $80 \%$ and $94 \%$, respectively, as we have previously reported [17]. Mo-DCs were obtained by a 5-d culture of monocytes in medium composed of $50 \mathrm{ng} / \mathrm{mL}$ GM-CSF (Peprotech) and $12 \mathrm{ng} / \mathrm{mL} \mathrm{IL-4} \mathrm{(Peprotech)} \mathrm{as} \mathrm{previously} \mathrm{reported} \mathrm{[18].} \mathrm{Fresh} \mathrm{complete} \mathrm{medium}$ was replaced in every 3 days. All blood samples from healthy donors were obtained in accordance with the 


\section{Cellular Physiology Cell Physiol Biochem 2016;40:499-508 \begin{tabular}{l|l|l|l} 
DOI: 10.1159/000452564 & (O) 2016 The Author(s). Published by S. Karger AG, Basel \\
and Biochemistry
\end{tabular}}

Shi et al: Inhibition of IL-23 in Human DCs by Cilostazo

National Institutes of Health approved institutional review board protocols.

Cells from the human embryonic kidney cell line HEK293T were incubated with DMEM medium containing $10 \%$ fetal bovine serum, $2 \mathrm{mM}$ L-Glutamine and $100 \mathrm{U} / \mathrm{ml}$ penicillin/streptomycin in an incubator with $\mathrm{CO}_{2}$ at $37^{\circ} \mathrm{C}$. HEK293T cells were seeded in 96-well plates overnight and were transfected according to the manufacturer's instructions.

\section{Cell Counting and Viability}

To determine whether cilostazol affects mo-DC cell counts and viability, the Guava ViaCount Assay was performed per the manufacturer's instructions.

\section{Dual-Luciferase Reporter Assay}

A luciferase assay was performed as previously reported [17]. In brief, the human IL23A (1 kbp fragment) was amplified from human genomic DNA and ligated into the pGL4.20 vector (Promega). HEK293T cells were seeded in 96-well plates overnight and then transfected with reporter plasmids encoding IL23A and plasmids for MyD88 using Lipofectamine 2000 (Invitrogen). Renilla luciferase expression vectors, pRLTK (Promega), were cotransfected as internal controls. To investigate the role of cilostazol on the MyD88induced $(20 \mathrm{ng})$ upregulation of IL23A promoter activity, different doses of cilostazol $(0-40 \mu \mathrm{M})$ or the solvent control (DMSO) were added as pretreatments at the beginning of transfection. Cell lysates were collected $40 \mathrm{~h}$ later, and luciferase activity was detected as previously described [17]. Luciferase units for IL23A were divided by the values for the renilla control.

ELISA

Levels of IL-23 in supernatants harvested from human mo-DCs were measured using an ELISA kit for human IL-23 from Mabtech according to the manufacturer's instructions.

\section{Real-Time PCR}

Total RNA was extracted from human mo-DCs using TRIzol reagents (Invitrogen, San Diego, CA) according to the manufacturer's instructions. IL-23 p19 and p40 gene expression levels were detected via the SYBR Green-based real-time PCR technique. The following primers specific for $\mathrm{p} 19, \mathrm{p} 40$ and EF1 $\alpha$ were shown as follows: for p19, sense 5'-CTC TGC TCC CTG ATA GCCCT-3' and antisense 5'-TGC GAA GGA TTT TGA AGCGG-3'; for p40, sense 5'-GGA GAG TCT GCC CAT TGAGG-3' and antisense 5'-TCT TGG GTG GGT CAG GTTTG-3'; for EF1 $\alpha$, sense $5^{\prime}$ - ATA TGG TTC CTG GCA AGCCC-3' and antisense 5'- GTG GGG TGG CAG GTA TTAGG-3'. PCRs were performed on a Corbett $65 \mathrm{H} 0$ machine (Corbett Research, Sidney, Australia) with the following cycling conditions: $95^{\circ} \mathrm{C}$ for $15 \mathrm{~s}$ and $60^{\circ} \mathrm{C}$ for $1 \mathrm{~min}$, for 40 cycles. The housekeeping gene EF $1 \alpha$ was used as the internal control. The quantification data were analyzed by Rotor-Gene Q Series Software 1.7 (Corbett Research, Sidney, Australia).

\section{Western Blotting}

Western blotting was performed as previously described [19]. Briefly, human mo-DCs were lysed in a buffer (1 mM each: antipain, benzamidine, leupeptin, pepstatin A, and phenylmethyl sulfonyl fluoride (PMSF), with $1 \%$ sodium dodecyl sulphate (SDS), and $5 \mathrm{mM}$ ethylene diamine tetraacetic acid (EDTA)) to obtain the total protein. Proteins (30 $\mu \mathrm{g}$ protein/lane) were electrophoresed on an 8\% SDS-polyacrylamide gel and transferred to nitrocellulose membranes (Pall Corporation, Ann Arbor, MI). After being blocked with BSA (3\% wt/vol) in tris-buffered saline containing Tween $20(0.5 \% \mathrm{vol} / \mathrm{vol}$; TBS-T) for $1 \mathrm{~h}$ at room temperature, the membranes were incubated with antibodies against p-AMPK (1:1000), AMPK (1:1000) and tubulin $(1: 3000)$ overnight at $4^{\circ} \mathrm{C}$. After rinsing three times, the blots were then incubated with the corresponding secondary antibodies at room temperature for $1 \mathrm{~h}$. Signals of bands were visualized by an enhanced chemiluminescence (ECL) detection system (Bio-Rad, Hercules, CA) and quantified using Quantity One Software (Bio-Rad).

\section{Statistical Analysis}

All data were presented as means \pm SEM. Significance of results was determined by one way ANOVA followed by Tukey post hoc testing using GraphPad Prism 5 software (GraphPad Software Inc., San Diego, CA, USA). $P$ value of less than 0.05 was accepted as statistically significant. 


\section{Results}

Cilostazol suppressed zymosan-induced IL-23 production in mo-DCs

To determine the effect of cilostazol on IL-23 production in mo-DCs, mo-DCs were pretreated with cilostazol $(10-40 \mu \mathrm{M})$ for $4 \mathrm{~h}$, and were then stimulated with zymosan $(20 \mu \mathrm{g} / \mathrm{ml})$ for $24 \mathrm{~h}$. ELISA data demonstrate that zymosan elicited IL-23 production, and cilostazol suppressed zymosan-induced IL-23 production in a concentration dependent fashion (Fig. 1A). In addition, as shown in Fig. 1B and 1C, the viability and number of mo-DCs were not obviously affected by the cilostazol treatment, which indicates that the inhibitory effect of cilostazol on IL-23 production was not due to negative effects on the viability and number of mo-DCs. Collectively, these data indicate that cilostazol inhibits the production of IL-23 at the protein level in human mo-DCs.

\section{Cilostazol suppressed IL-23 $p 19$ and $p 40$ expression}

IL-23 is a heterodimer composed of the subunits p19 and p40. RT-PCR analyses were performed to determine whether cilostazol affects IL-23 production at the transcription level. As shown in Fig. 2, there was an up-regulation of both p19 and p40 subunit expression upon zymosan stimulation. More importantly, it was demonstrated that cilostazol exhibited a significant inhibitory effect on the subunits $\mathrm{p} 19$ and p40 (Fig. 2) that was not demonstrated in the solvent control DMSO. These results suggest that cilostazol inhibits IL-23 production at the RNA level in human mo-DCs. In combination with the aforementioned ELISA data, these results indicate that cilostazol inhibits IL-23 production, resulting in decreased IL-23 protein and RNA levels in human mo-DCs.

\section{Cilostazol inhibited MyD88-induced IL23A transcriptional activity}

Luciferase reporter assay was utilized to determine the effect of cilostazol on IL23A transcriptional activity in HEK293T cells. As shown in Fig. 3, IL23A promoter activity was

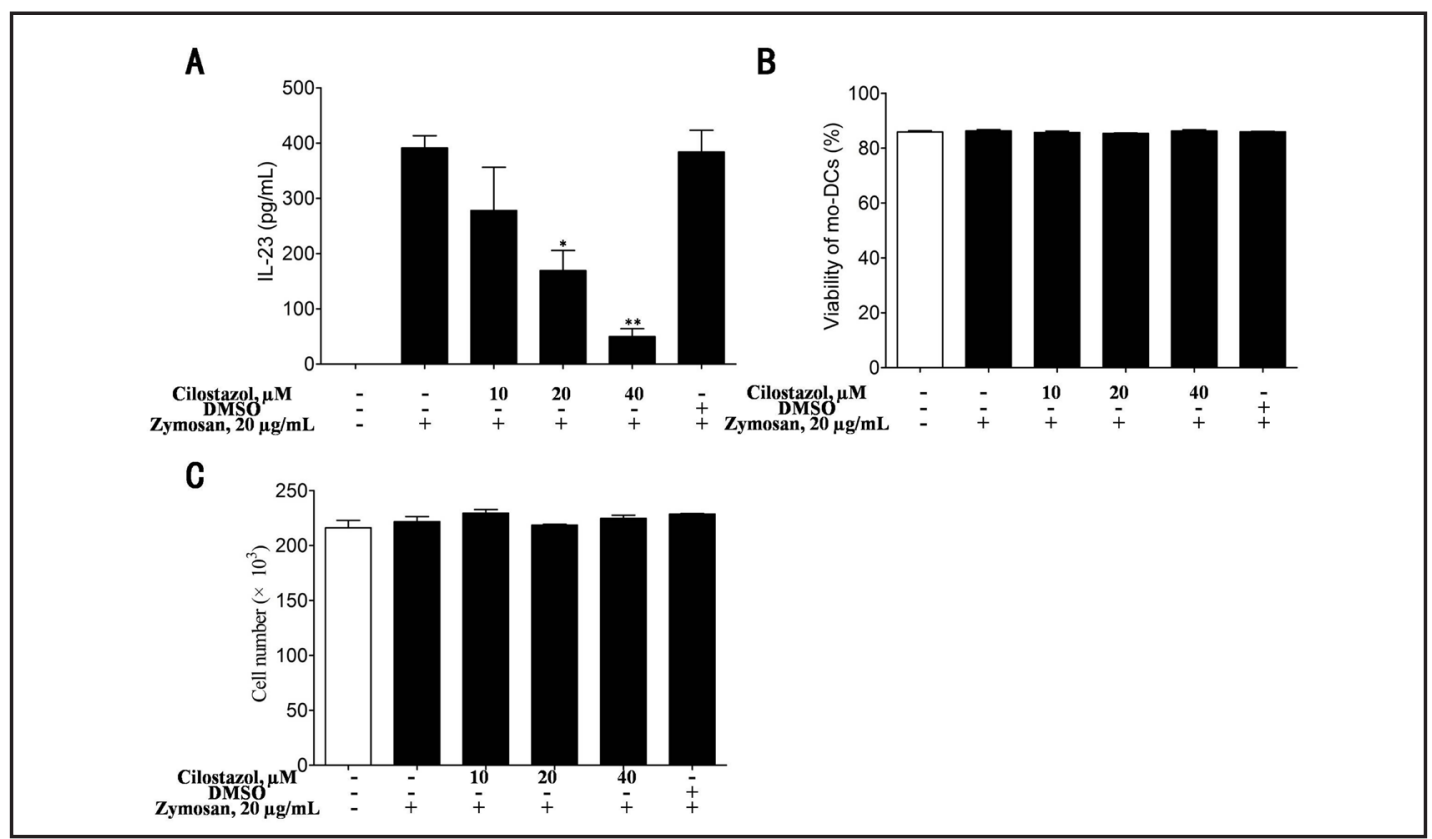

Fig. 1. Influence of cilostazol on IL-23 expression, cell count and viability in human mo-DCs. (A, B and C) Mo-DCs were pretreated for $4 \mathrm{~h}$ with cilostazol $(0-40 \mu \mathrm{M})$ or the solvent control (DMSO). Cells were then incubated with zymosan $(20 \mu \mathrm{g} / \mathrm{ml})$ in the presence or absence of cilostazol for $24 \mathrm{~h}$. (A) Expression levels of IL-23 protein were determined by ELISA $24 \mathrm{~h}$ after stimulation. (B and C) Cell counts and viability of moDCs were detected via the Guava ViaCount Assay. Data represent the mean \pm SEM of 3 separate experiments. ${ }^{*} P<0.05$ vs. zymosan alone, ${ }^{* *} P<0.01$ vs. zymosan alone. 


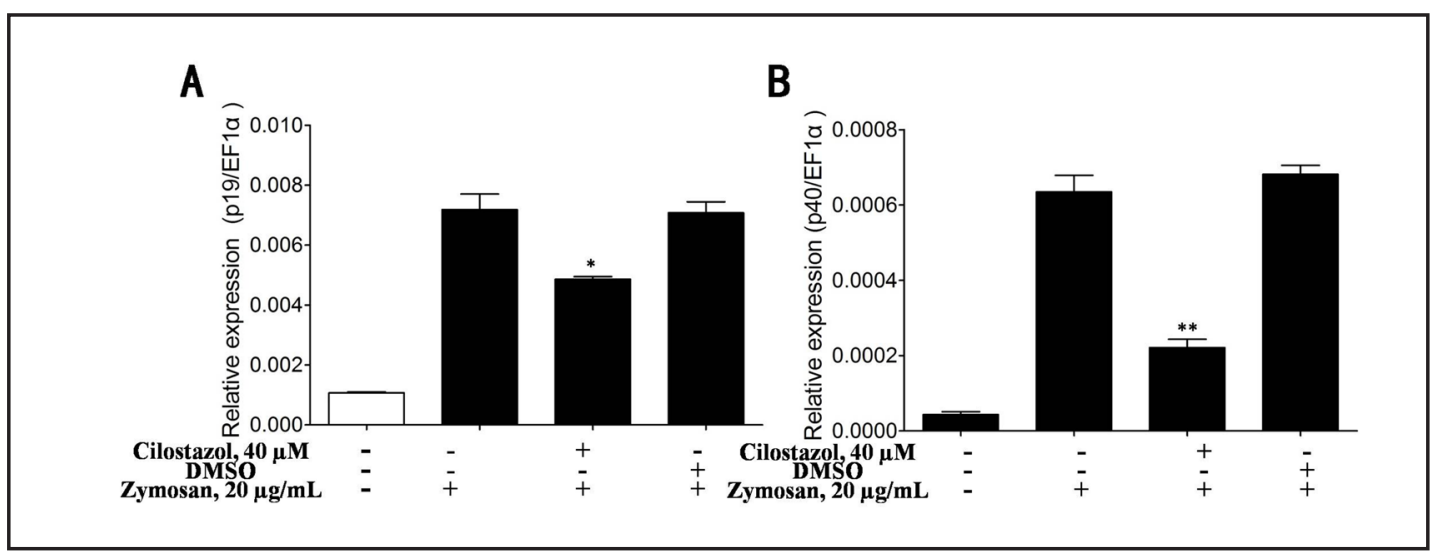

Fig. 2. Influence of cilostazol on IL-23 p19 and p40 subunits in mo-DCs. Mo-DCs were pretreated for $4 \mathrm{~h}$ with cilostazol $(40 \mu \mathrm{M})$ or the solvent control (DMSO). Cells were then incubated with zymosan $(20 \mu \mathrm{g} / \mathrm{ml})$ in the presence or absence of cilostazol for $5 \mathrm{~h}$. Expression of (A) IL-23 p19 and (B) IL-23 p40 was analyzed via RT-PCR 5 hours after stimulation. Data represent the mean \pm SEM of 3 separate experiments. ${ }^{*} P<0.05$ vs. zymosan alone, ${ }^{* *} P<0.01$ vs. zymosan alone.

Fig. 3. Influence of cilostazol on MyD88-induced IL23A promoter activity in HEK293T cells. MyD88 (20 ng) was cotransfected with IL-23 p19 (100 ng) in the presence or absence of different doses of cilostazol (0-40 $\mu \mathrm{M})$, and the IL23A promoter activity was then assayed. In brief, after being pretreated with cilostazol (0-40 $\mu \mathrm{M})$ or DMSO for $1 \mathrm{~h}$, HEK293T cells were transfected with the indicated plasmids using Lipofectamine 2000. Luciferase units of IL23A promoter activity were divided by their renilla control values. Data are representative of 3 separate experiments. ${ }^{*} P<0.05$ vs. no treatment, ${ }^{* *} P<0.01$ vs. no treatment.



Fig. 4. The effect of A-769662 and Compound C on IL-23 protein expression in zymosan-activated Mo-DCs. Mo-DCs were pretreated for 4 h with cilostazol $(40 \mu \mathrm{M})$, the solvent control (DMSO), A-769662 (0-100 $\mu \mathrm{M})$ and/or Compound $C(50 \mu \mathrm{M})$. Cells were then incubated with zymosan $(20 \mu \mathrm{g} / \mathrm{ml})$ for $24 \mathrm{~h}$. Expression of the IL-23 protein was analyzed by ELISA 24 hours after stimulation. Data represent the mean \pm SEM of 3 separate experiments. ${ }^{*} P<0.05$ vs. zymosan alone, ${ }^{* *} P<0.01$ vs. zymosan alone, ${ }^{\#} P<0.05$ vs. cilostazol plus zymosan treatment.



significantly increased upon MyD88 transfection. More importantly, cilostazol (0-40 $\mu \mathrm{M}$ ) led to a down-regulation of MyD88-induced IL23A transcriptional activity in a concentration dependent manner, whereas the solvent control DMSO did not (Fig. 3). The results suggest that cilostazol suppresses MyD88-mediated IL23A promoter activity.

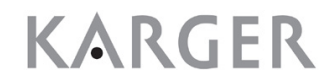


Fig. 5. Influence of cilostazol on the expression and phosphorylation of AMPK in moDCs. Mo-DCs were pretreated for $4 \mathrm{~h}$ with cilostazol $(0-40 \mu \mathrm{M})$ or the solvent control (DMSO). Cells were then incubated with zymosan $(20 \mu \mathrm{g} / \mathrm{ml})$ in the presence or absence of cilostazol for $1 \mathrm{~h}$. Expression and phosphorylation of AMPK were detected via Western blotting. Data represent the mean \pm SEM of 3 separate experiments. ${ }^{* *} P<0.01$ vs. zymosan alone.

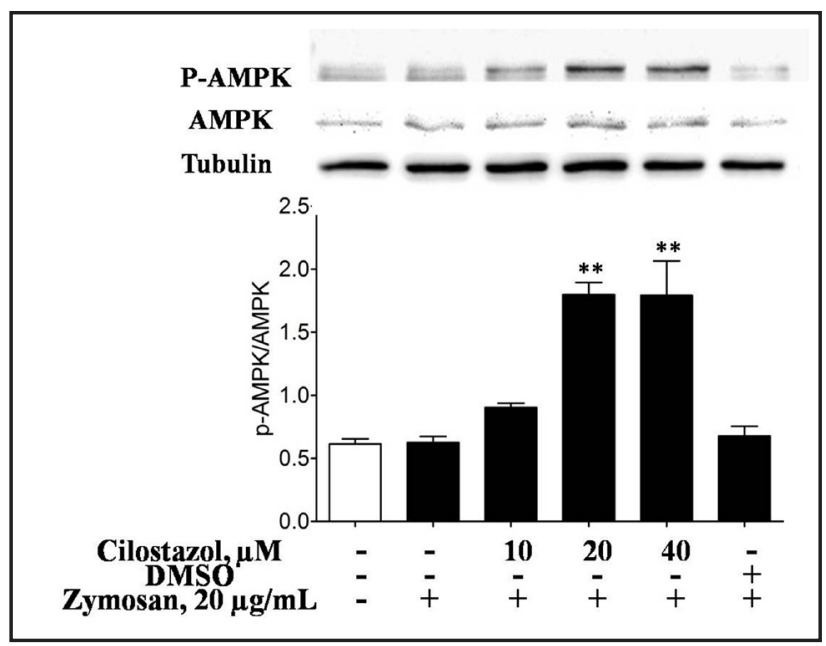

Cilostazol can mimic the effect of the AMPK agonist A-769662 on IL-23 production, and the effect of cilostazol on IL-23 production can be blocked by the AMPK antagonist Compound C

It has been reported that AMPK pathways are involved in cilostazol's mechanism of action $[20,21]$. Thus, we aimed to clarify whether the inhibitory effect of cilostazol on IL-23 production by mo-DCs was mediated by AMPK by treating cells with an AMPK agonist (A769662) and an AMPK antagonist (Compound C). As shown in Fig. 4, A-769662 acted in a concentration dependent manner to suppress the production of IL-23 in a manner similar to that of cilostazol. More importantly, Compound $\mathrm{C}$ was able to block the effect of cilostazol (Fig. 4). The present study strongly suggests that cilostazol exerts its inhibitory effect on IL23 production in human mo-DCs, at least partially, via an AMPK-dependent pathway.

Cilostazol promoted the phosphorylation level of AMPK in mo-DCs upon zymosan stimulation

The aforementioned results indirectly indicate that the inhibitory effect of cilostazol on IL-23 is related to the AMPK pathway. To further clarify the underlying mechanisms, we directly measured the expression and phosphorylation of AMPK. As shown in Fig. 5, cilostazol effectively promoted the phosphorylation of AMPK in mo-DCs, indicating that cilostazol may suppress the IL-23 production in mo-DCs via the activation of AMPK.

\section{Discussion}

In the present study, treatment with cilostazol was demonstrated to suppress IL-23 production via the activation of AMPK in human mo-DCs. This conclusion is based on the following observations: 1) cilostazol inhibited zymosan-induced IL-23 production at both the protein and RNA levels, as well as MyD88-induced IL23A promoter activity; 2) cilostazol mimicked the effect of an AMPK agonist (A-769662) on IL-23 production, and the effect of cilostazol on IL-23 production can be blocked by an AMPK antagonist (Compound C); and 3) cilostazol promoted the phosphorylation of AMPK.

DCs, important IL-23 producing cells in the human body, play a pivotal role in innate and adaptive immune responses $[3,4]$ and have received attention as promising targets for treating coronary heart disease and other cardiovascular diseases [22, 23]. In the present study, we have demonstrated for the first time that cilostazol suppresses IL-23 production in human mo-DCs, suggesting that cilostazol is able to regulate DC function. However whether cilostazol could be used as a potential drug to target DCs warrants further study.

Some reports have shown that the effect of cilostazol is associated with the activation of the AMPK pathway, and this effect has been validated in various models [20, 21, 24, 25]. 
Furthermore, AMPK has been suggested to play an important role in TLRs-induced IL-23 production [26]. However, it remains unclear whether the inhibitory effect of cilostazol on IL-23 production is mediated via an AMPK-dependent pathway in human mo-DCs. In the present study, it was observed that cilostazol had the same effect as an AMPK agonist (A769662) on IL-23 production, and the effect of cilostazol on IL-23 production was blocked by an AMPK antagonist (Compound C), suggesting that the underlying mechanism of the cilostazol-induced inhibitory effect on IL-23 was mediated via an AMPK pathway. Moreover, the Western blotting analysis showed that cilostazol promoted the phosphorylation of AMPK in mo-DCs. Collectively, our data demonstrate that cilostazol suppresses IL-23 production via the activation of an AMPK-dependent pathway in human mo-DCs. However, in the present study, we used human IL-23A gene promoter ( $1 \mathrm{~kb}$ fragment), which includes $\mathrm{\kappa B}$, $\mathrm{C} / \mathrm{EBP} \beta, \mathrm{ATF} 2[27,28]$. It has been reported that AMPK-IK $\beta \alpha-\mathrm{NF} \kappa \mathrm{B}$ signaling is involved in sildenafil's anti-inflammatory effects [29]. In addition, it has been reported that AMPK could suppressed C/EBP $\beta$ expression [30, 31]. As to ATF2, it has been reported that AMPK could phosphorylate ATF1, but not ATF2 [32]. And the downstream transcription factors affected by AMPK, which contribute to IL-23 suppression, need to be further characterized.

While our results are the first to demonstrate cilostazol's anti-inflammatory properties in human mo-DCs, numerous studies have reported these properties in other cell-types. Shin et al. found that cilostazol suppresses NAD(P)H oxidase-dependent superoxide formation and inflammatory cytokine production in HUVECs (human umbilical vein endothelial cells) [11]. In addition, it has been reported that cilostazol inhibits the expression of adhesion molecules and chemokine release by HUVECs [12], indicating that cilostazol has an anti-inflammatory effect on HUVECs. Furthermore, it has been demonstrated that the anti-inflammatory effects of cilostazol may result from cAMP-dependent protein kinase activation-mediated inhibition of NF- $\kappa B$ gene transcription [13]. Cilostazol has also been shown to inhibit IFN- $\alpha$ and TNF- $\alpha$ production from plasmacytoid dendritic cells in a dosedependent manner [14]. In addition, cilostazol has been reported to suppress TNF- $\alpha$ and IL-1 $\beta$ production in synovial macrophages from RA patients via inhibition of TLR4/MyD88/ NF- $\kappa B$ signaling pathways [15]. Moreover, cilostazol inhibits TLR2-induced IL-23 production in human synovial macrophages by suppressing the RhoA pathway via a cAMP-dependent pathway [16]. These studies suggest that cilostazol serves as a potent anti-inflammatory drug with immunomodulatory properties, and our study further demonstrates the pleiotropic effects of cilostazol.

This study does have some limitations. In the present study, zymosan stimulation was demonstrated to be a strong inducer of IL-23 production in mo-DCs. It has been demonstrated that zymosan triggers signal transduction through dectin-1 and TLR2 and that it activates signal cascades, including ERK and p38 MAPK, and these proteins are involved in zymosan-induced signaling pathways [27]. It remains unclear whether cilostazol suppresses IL-23 production in mo-DCs by regulating the phosphorylation level of ERK and p38 MAPK after a zymosan challenge. Signaling pathways regarding IL-23 production in mo-DCs are interconnected, so the present study cannot preclude the possibility that other mechanisms may be involved. In addition, the concentration of cilostazol used in the present study was far greater than its serum concentrations (approximately $2 \mu \mathrm{M}$ ) in the human body. However, considering the intestinal absorption of cilostazol, it can be posited that local serum cilostazol concentrations in the small intestine are obviously far greater than the blood concentration in the body. More importantly, it has been validated by Akiyama et al. that the local concentration in the stomach, small intestine and liver can be 21, 4 and 4 times higher than the blood concentration [33]. Based on this observation, it seems that the roles of cilostazol in intestinal inflammation should be considered and carefully evaluated in the future. This raises the question as to whether cilostazol influences IL-23 production in clinical practice. Future clinical studies are warranted to clarify whether the beneficial effect of cilostazol in clinic practice may be related to its inhibitory effect on IL-23 production. In addition, the inhibition of IL-23 protein by cilostazol is higher than the inhibition of both p19 and p40 gene expression. This phenomenon can be explained by the following 
reasons. First, p19 and p40 both are affected by cilostazol at the RNA level, and this may contribute to the significant inhibition of IL-23 at protein level; second, it has been reported that AMPK suppresses [34, 35] or increases [36, 37] mRNA stability. The opposing effect of AMPK activators on mRNA stability in different cell types may be explained by cell-type specific cross-talk between the AMPK and other kinase cascades [38]. Therefore, we could not preclude the possibility that cilostazol and subsequent activation of AMPK in human DCs may have impacts on IL-23 mRNA stability and/or translation. In addition, tristetraprolin has been demonstrated to decrease the stability of IL-23 mRNA and the expression level of IL-23 $[39,40]$. This raises the question as to whether cilostazol affects tristetraprolin?

In conclusion, we have demonstrated for the first time that cilostazol suppresses zymosan-induced IL-23 production in human mo-DCs via an AMPK-dependent pathway. These results not only indicate a novel mechanism for the anti-inflammatory role of cilostazol but also suggest a potential role for cilostazol in the treatment of IL-23 related diseases.

\section{Acknowledgments}

The authors thank Mr George Linderman for his invaluable help in editing and polishing the English language and grammar of this manuscript. This work was supported by research grants from the Key Research Project of the 306th Hospital of PLA in 2013 (No. 13ZD01).

\section{Disclosure Statement}

The authors have declared no conflicts of interest.

\section{References}

1 Dawson DL, Cutler BS, Meissner MH, Strandness DE Jr: Cilostazol has beneficial effects in treatment of intermittent claudication: Results from a multicenter, randomized, prospective, double-blind trial. Circulation 1998;98:678-686.

2 Kambayashi J, Liu Y, Sun B, Shakur Y, Yoshitake M, Czerwiec F: Cilostazol as a unique antithrombotic agent. Curr Pharm Des 2003;9:2289-2302.

3 Brombacher F, Kastelein RA, Alber G: Novel IL-12 family members shed light on the orchestration of Th1 responses. Trends Immunol 2003;24:207-212.

4 Trinchieri G, Pflanz S, Kastelein RA: The IL-12 family of heterodimeric cytokines: New players in the regulation of T cell responses. Immunity 2003;19:641-644.

5 McKenzie BS, Kastelein RA, Cua DJ: Understanding the IL-23-IL-17 immune pathway. Trends Immunol 2006;27:17-23.

6 Langrish CL, McKenzie BS, Wilson NJ, de Waal Malefyt R, Kastelein RA, Cua DJ: IL-12 and IL-23: Master regulators of innate and adaptive immunity. Immunol Rev 2004;202:96-105.

7 Murphy CA, Langrish CL, Chen Y, Blumenschein W, McClanahan T, Kastelein RA, Sedgwick JD, Cua DJ: Divergent pro- and antiinflammatory roles for IL-23 and IL-12 in joint autoimmune inflammation. J Exp Med 2003;198:1951-1957.

8 An J, Li T, Dong Y, Li Z, Huo J: Terminalia chebulanin attenuates psoriatic skin lesion via regulation of heme oxygenase-1. Cell Physiol Biochem 2016;39:531-543.

9 Subramanian M, Thorp E, Tabas I: Identification of a non-growth factor role for GM-CSF in advanced atherosclerosis: Promotion of macrophage apoptosis and plaque necrosis through IL-23 signaling. Circ Res 2015;116:e13-24.

$10 \mathrm{Hu}$ X, Ma R, Lu J, Zhang K, Xu W, Jiang H, Da Y: IL-23 promotes myocardial I/R injury by increasing the inflammatory responses and oxidative stress reactions. Cell Physiol Biochem 2016;38:2163-2172.

11 Shin HK, Kim YK, Kim KY, Lee JH, Hong KW: Remnant lipoprotein particles induce apoptosis in endothelial cells by NAD(P)H oxidase-mediated production of superoxide and cytokines via lectin-like oxidized lowdensity lipoprotein receptor-1 activation: Prevention by cilostazol. Circulation 2004;109:1022-1028. 


\section{Cellular Physiology Cell Physiol Biochem 2016;40:499-508 \begin{tabular}{l|l|l} 
and BOI: 10.1159/000452564 & $\begin{array}{l}\text { (c) 2016 The Author(s). Published by S. Karger AG, Basel } \\
\text { www.karger.com/cpb- }\end{array}$
\end{tabular}}

Shi et al.: Inhibition of IL-23 in Human DCs by Cilostazol

12 Park SY, Lee JH, Kim YK, Kim CD, Rhim BY, Lee WS, Hong KW: Cilostazol prevents remnant lipoprotein particle-induced monocyte adhesion to endothelial cells by suppression of adhesion molecules and monocyte chemoattractant protein-1 expression via lectin-like receptor for oxidized low-density lipoprotein receptor activation. J Pharmacol Exp Ther 2005;312:1241-1248.

13 Park SY, Lee JH, Kim CD, Lee WS, Park WS, Han J, Kwak YG, Kim KY, Hong KW: Cilostazol suppresses superoxide production and expression of adhesion molecules in human endothelial cells via mediation of cAMP-dependent protein kinase-mediated maxi-K channel activation. J Pharmacol Exp Ther 2006;317:1238-1245.

14 Sun F, Yin Z, Yu HS, Shi QX, Zhao B, Zhang LG, Wang SL: Cilostazol inhibits plasmacytoid dendritic cell activation and antigen presentation. J Geriatr Cardiol 2015;12:388-393.

15 Park SY, Lee SW, Baek SH, Lee CW, Lee WS, Rhim BY, Hong KW, Kim CD: Suppression of PU.1-linked TLR4 expression by cilostazol with decrease of cytokine production in macrophages from patients with rheumatoid arthritis. Br J Pharmacol 2013;168:1401-1411.

16 Park SY, Lee SW, Lee WS, Rhim BY, Lee SJ, Kwon SM, Hong KW, Kim CD: RhoA/ROCK-dependent pathway is required for TLR2-mediated IL-23 production in human synovial macrophages: Suppression by cilostazol. Biochem Pharmacol 2013;86:1320-1327.

17 Shi Q Yin Z, Zhao B, Sun F, Yu H, Yin X, Zhang L, Wang S: PGE2 elevates IL-23 production in human dendritic cells via a cAMP dependent pathway. Mediators Inflamm 2015;2015:984690.

18 Gantner BN, Simmons RM, Canavera SJ, Akira S, Underhill DM: Collaborative induction of inflammatory responses by dectin-1 and Toll-like receptor 2. J Exp Med 2003;197:1107-1117.

19 Shi QX, Zhang LJ, Yao Y, Zhang QY, Wang W, Li J, Shang YL, Bi H, Zhang SM, Guo HT, Wang YM, Yu SQ, Yi DH, Bueno FR, Kaye AD, Pei JM: Kappa-opioid receptor activation prevents against arrhythmias by preserving cx43 protein via alleviation of intracellular calcium. Am J Ther 2013;20:493-501.

20 Motta NA, de Brito FC: Cilostazol exerts antiplatelet and anti-inflammatory effects through AMPK activation and NFKB inhibition on hypercholesterolaemic rats. Fundam Clin Pharmacol 2016;30:327-337.

21 Tseng SY, Chao TH, Li YH, Liu PY, Lee CH, Cho CL, Wu HL, Chen JH: Cilostazol improves high glucoseinduced impaired angiogenesis in human endothelial progenitor cells and vascular endothelial cells as well as enhances vasculoangiogenesis in hyperglycemic mice mediated by the adenosine monophosphateactivated protein kinase pathway. J Vasc Surg 2016;63:1051-1062.e1053.

22 Dieterlen MT, John K, Reichenspurner H, Mohr FW, Barten MJ: Dendritic cells and their role in cardiovascular diseases: a view on human studies. J Immunol 2016;2016:5946807.

23 Zhang Y, Zhang C: Role of dendritic cells in cardiovascular diseases. World J Cardiol 2010;2:357-364.

24 Chang KC: Cilostazol inhibits HMGB1 release in LPS-activated RAW 264.7 cells and increases the survival of septic mice. Thromb Res 2015;136:456-464.

25 Kim JE, Sung JY, Woo CH, Kang YJ, Lee KY, Kim HS, Kwun WH, Choi HC: Cilostazol inhibits vascular smooth muscle cell proliferation and reactive oxygen species production through activation of AMP-activated protein kinase induced by heme oxygenase-1. Korean J Physiol Pharmacol 2011;15:203-210.

26 Alvarez Y, Rodriguez M, Municio C, Hugo E, Alonso S, Ibarrola N, Fernandez N, Crespo MS: Sirtuin 1 is a key regulator of the interleukin-12 p70/interleukin-23 balance in human dendritic cells. J Biol Chem 2012;287:35689-35701.

27 Rodriguez M, Domingo E, Alonso S, Frade JG, Eiros J, Crespo MS, Fernandez N: The unfolded protein response and the phosphorylations of activating transcription factor 2 in the trans-activation of il23a promoter produced by beta-glucans. J Biol Chem 2014;289:22942-22957.

28 Rodriguez M, Marquez S, Montero O, Alonso S, Frade JG, Crespo MS, Fernandez N: Pharmacological inhibition of eicosanoids and platelet-activating factor signaling impairs zymosan-induced release of IL-23 by dendritic cells. Biochem Pharmacol 2016;102:78-96.

29 Nunes AK, Raposo C, Rocha SW, Barbosa KP, Luna RL, da Cruz-Hofling MA, Peixoto CA: Involvement of AMPK, IK $\beta \alpha-N F \kappa B$ and eNOS in the sildenafil anti-inflammatory mechanism in a demyelination model. Brain Res 2015;1627:119-133.

30 Matsuda T, Takahashi H, Mieda Y, Shimizu S, Kawamoto T, Matsuura Y, Takai T, Suzuki E, Kanno A, KoyanagiKimura M, Asahara S, Bartolome A, Yokoi N, Inoue H, Ogawa W, Seino S, Kido Y: Regulation of pancreatic $\beta$ cell mass by cross-interaction between CCAAT Enhancer Binding Protein $\beta$ induced by endoplasmic reticulum stress and AMP-activated protein kinase activity. PLoS One 2015;10:e0130757. 


\section{Cellular Physiology Cell Physiol Biochem 2016;40:499-508

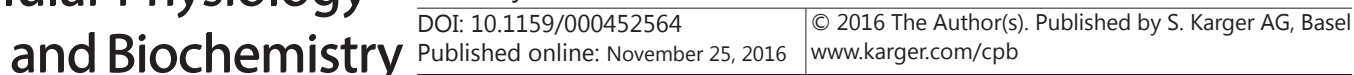

Shi et al.: Inhibition of IL-23 in Human DCs by Cilostazol

31 Choudhury M, Qadri I, Rahman SM, Schroeder-Gloeckler J, Janssen RC, Friedman JE: C/EBP $\beta$ is AMP kinase sensitive and up-regulates PEPCK in response to ER stress in hepatoma cells. Mol Cell Endocrinol 2011;331:102-108.

32 Thomson DM, Herway ST, Fillmore N, Kim H, Brown JD, Barrow JR, Winder WW: AMP-activated protein kinase phosphorylates transcription factors of the CREB family. J Appl Physiol (1985) 2008;104:429-438.

33 Akiyama H, Kudo S, Shimizu T: The absorption, distribution and excretion of a new antithrombotic and vasodilating agent, cilostazol, in rat, rabbit, dog and man. Arzneimittelforschung 1985;35:1124-1132.

34 Zhang W, Wang Q Wu Y, Moriasi C, Liu Z, Dai X, Wang Q Liu W, Yuan ZY, Zou MH: Endothelial cell-specific liver kinase B1 deletion causes endothelial dysfunction and hypertension in mice in vivo. Circulation 2014;129:1428-1439.

35 Wang W, Fan J, Yang X, Furer-Galban S, Lopez de Silanes I, von Kobbe C, Guo J, Georas SN, Foufelle F, Hardie DG, Carling D, Gorospe M: AMP-activated kinase regulates cytoplasmic HuR. Mol Cell Biol 2002;22:34253436.

36 Li D, Zhang Y, Ma J, Ling W, Xia M: Adenosine monophosphate activated protein kinase regulates ABCG1mediated oxysterol efflux from endothelial cells and protects against hypercholesterolemia-induced endothelial dysfunction. Arterioscler Thromb Vasc Biol 2010;30:1354-1362.

37 Ouchi N, Shibata R, Walsh K: AMP-activated protein kinase signaling stimulates VEGF expression and angiogenesis in skeletal muscle. Circ Res 2005;96:838-846.

38 Eberhardt W, Doller A, Akool el S, Pfeilschifter J: Modulation of mRNA stability as a novel therapeutic approach. Pharmacol Ther 2007;114:56-73.

39 Molle C, Zhang T, Ysebrant de Lendonck L, Gueydan C, Andrianne M, Sherer F, Van Simaeys G, Blackshear PJ, Leo O, Goriely S: Tristetraprolin regulation of interleukin 23 mRNA stability prevents a spontaneous inflammatory disease. J Exp Med 2013;210:1675-1684.

40 Lee HH, Yang SS, Vo MT, Cho WJ, Lee BJ, Leem SH, Lee SH, Cha HJ, Park JW: Tristetraprolin down-regulates IL-23 expression in colon cancer cells. Mol Cells 2013;36:571-576. 